\title{
¿Cómo es el suelo de nuestro huerto? El Aprendizaje Basado en Problemas como estrategia en Educación Ambiental desde el Grado de Maestro/a en Educación Infantil
}

\section{How is the soil in our garden? Problem Based Learning in Environmental Education as a strategy from the Degree of Preschool Teacher Training}

\section{Lourdes Aragón Núñez Isabel María Cruz Lorite}

Universidad de Cádiz

Resumen: En el siguiente trabajo se diseña, desarrolla y valora una propuesta educativa a través de la estrategia de Aprendizaje Basado en Problemas (ABP) en la asignatura de Educación Ambiental en Infantil. El ABP permite desarrollar en los estudiantes de $4^{\circ}$ curso del Grado en Educación Infantil durante el curso 2014/2015, habilidades y competencias fundamentales en su futuro profesional, como el trabajo en equipo o la resolución de problemas a la vez que indagan sobre sus propios conocimientos en torno al tema del suelo. En esta propuesta se utiliza el huerto ecológico para contextualizar y dar sentido al ABP a través de la resolución de un problema real al que los estudiantes deben dar solución.

Palabras clave: ABP, competencias, Educación Ambiental, formación inicial de maestros/as, huerto

Abstract: This article, is aiming to a design, develop and evaluate educational proposal through strategy Problem-Based Learning (PBL) in the subject of Preschool Environmental Education. PBL allows students from 4th year of the Degree of Preschool Teacher Training during the course 2014/2015, developing skills and competences such as teamwork and problem solving as well as inquiring about their own knowledge about the soil. In this proposal, the organic garden is used as a context and gives meaning to PBL by challenging students to solve a real problem.

Keywords: Competences, Environmental Education, Teacher Training, Garden, PBL

(Fecha de recepción: julio, 2015, y de aceptación: abril, 2016)

DOI: 10.7203/DCES.30.6475 


\section{Introducción}

En el ámbito de la Educación Ambiental (EA) existe una enorme confusión respecto a los modelos educativos que orientan el proceso de enseñanza-aprendizaje. Como señala García (2004), la EA se trata de un ámbito de pensamiento y acción en el que predomina la heterogeneidad y el debate, $y$ en el que existen diversidad de paradigmas teóricos, estrategias, practicantes y escenarios. Lejos de una EA de corte ambientalista, la cual ha derivado, frecuentemente, a una perspectiva psicoeducativa tecnicista y conductista, en la que se pretende desarrollar actitudes y destrezas proambientales sin atender a lo que saben los propios sujetos que aprenden, el modelo que se pretende trabajar desde la titulación del Grado de Maestro/a en Educación Infantil es el de una EA dirigida a la capacitación para la acción. Un modelo con múltiples variantes y submodelos, próximo al desarrollo sostenible y al cambio social (García, 2002). Para ello, se hace necesario indagar en otras metodologías más próximas a la perspectiva constructivista, o bien en metodologías que consideran al alumno parte activa de su propio aprendizaje.

En este contexto, el Aprendizaje Basado en Problemas (ABP), podría ser una buena alternativa para desarrollar en nuestros alumnos competencias que les permitan adquirir conocimientos necesarios para proponer alternativas a los problemas ambientales desde una visión investigativa, a la vez, que se potencia en ellos una concienciación ambiental, la reflexión y el análisis crítico a los problemas ambientales y a las estrategias de intervención.

El ABP se trata de una metodología muy acorde a los fines educativos establecidos en el marco del Espacio Europeo de Educación Superior (EEES), que ha supuesto importantes cambios en el modelo de enseñanza-aprendizaje (Martín-Peña, Díaz-Garrido y SánchezLópez, 2015). Por otra parte, como señalan González y Castro (2011) uno de los retos que los docentes universitarios presentan en la formación de competencias profesionales, es que se les exige el diseño de escenarios educativos que promuevan experiencias de aprendizaje que permitan a las personas enfrentar con éxito problemas relevantes en el campo de su formación profesional. En base a esto, el ABP también podría ser una alternativa adecuada, ya que permite diseñar problemas a partir de situaciones reales o simuladas, relacionadas con la construcción del conocimiento o el ejercicio reflexivo de una determinada destreza en el ámbito de un conocimiento, práctica o ejercicio profesional (González y Castro, 2011). Sin embargo, esto no siempre se logra, y una de las limitaciones que presenta esta estrategia es precisamente que en muchas ocasiones las situaciones o problemas que se utilizan están descontextualizadas, siendo artificiales y poco vinculadas con la acción en contextos reales (Esteban, 2011).

En este sentido, en el presente trabajo se pretende diseñar, desarrollar y 
valorar una propuesta didáctica a través de la metodología de $A B P$, y en el que se utiliza el Huerto Ecológico Universitario (HEU) como escenario educativo, con objeto de acercar, contextualizar y dar sentido a la estrategia llevada a cabo desde la asignatura de Educación Ambiental en Infantil (EAI) en el Grado de Maestro/a en Educación Infantil. Este trabajo se centra en des- cribir y analizar la primera fase de las cuatro que se estructura la asignatura y que da sentido al HEU (tabla I). La fase 4 , se trata de acciones que tienen lugar a lo largo de las otras tres fases ya que supone el diseño, la gestión, y el desarrollo en definitiva, del propio HEU, a medida que se van realizando las otras fases que componen la asignatura.

\section{Tabla I \\ Estructura de la asignatura de EAI en base al ABP utilizando como contexto educativo el HEU}

(Fuente: Elaboración propia)

\begin{tabular}{|c|c|c|}
\hline Fase I & $\begin{array}{l}\text { Desarrollo de la estrategia ABP en el contexto del HEU: } \\
\text { ¿cómo es el suelo de nuestro huerto?¿es cultivable? }\end{array}$ & \multirow{3}{*}{$\begin{array}{l}\text { Fase IV } \\
\text { Diseño, gestión y ta- } \\
\text { reas propias para lle- } \\
\text { var a cabo el huerto } \\
\text { ecológico universita- } \\
\text { rio a través del traba- } \\
\text { jo cooperativo }\end{array}$} \\
\hline Fase II & $\begin{array}{l}\text { Del suelo a un problema ambiental: la erosión } \\
\text { Experiencia realizada en el laboratorio para trabajar un proble- } \\
\text { ma ambiental como es la erosión del suelo } \\
\text { Experiencia como referencia para diseñar y desarrollar acciones } \\
\text { didácticas en la etapa de infantil en torno a un problema ambien- } \\
\text { tal vinculado al huerto ecológico. }\end{array}$ & \\
\hline Fase III & $\begin{array}{l}\text { El Huerto de las Ciencias: diseño, desarrollo y valoración de talle- } \\
\text { res en cuatro aulas de niños y niñas de } 4 \text { y } 5 \text { años en torno a un } \\
\text { problema ambiental: } \\
\text { a) Investigar sobre un problema ambiental que pueda ser } \\
\text { abordado desde los recursos del huerto } \\
\text { b) Diseño de un plan de acción para llevar a cabo un taller } \\
\text { con objeto de acercar a los niños y niñas a un problema } \\
\text { ambiental } \\
\text { c) Desarrollo de las propuestas en el centro escolar } \\
\text { d) Valorar la práctica educativa (docente, alumnos y tutores } \\
\text { del centro escolar) }\end{array}$ & \\
\hline
\end{tabular}

\section{Marco teórico y fundamentación}

La implementación de la EEES implica un cambio en la organización del curriculum y en las metodologías didácticas empleadas en los centros de enseñanza superior. El ABP se presenta como una propuesta posible para afrontar el cambio en el contexto universitario, y concretamente en la formación de los futuros maestros/as, ya que constituye no sólo un método de 
enseñanza activo centrado en el alumno sino también un enfoque curricular que cambia la organización tradicional del currículum en base a disciplinas por otra basada en problemas (Egido et al., 2007).

El ABP es una metodología de aprendizaje inductivo en la que el alumno se convierte en el protagonista de su propio aprendizaje. Los estudiantes trabajan en pequeños grupos y son tutorizados por el profesor; utilizan la resolución de un problema como base para alcanzar unos objetivos de aprendizaje y desarrollar unas competencias y habilidades transferibles a la práctica profesional (Gómez-Esquer, Rivas, Mercado y Barjola, 2009). Como punto de partida del proceso didáctico se usa un problema similar al que el estudiante podrá encontrarse en el futuro durante su ejercicio profesional, lo que permite activar los conocimientos previos de los estudiantes, explicitar lo que saben y lo que no para resolverlo y detectar sus propias necesidades de aprendizaje (Egido et al., 2007).

Se trata de una estrategia de aprendizaje en la que el estudiante toma un papel activo, de indagador critico independiente, dueño de sus propias experiencias de aprendizaje, mientras que el profesor debe provocar oportunidades de aprendizaje, escuchando al alumnado, promoviendo el trabajo en grupo, formulando las preguntas adecuadas para encarrilar el trabajo en el grupo u orientar en el uso de las fuentes de información (Solaz-Portolés, Sanjosé y Gómez, 2011). Como señala Manza- nares (2008) muchas de las características que configuran el ABP tienen su base teórica en la psicología cognitiva, en concreto, en la visión constructivista del aprendizaje. Así, esta metodología se sustenta en principios constructivistas tales como que la comprensión de una situación de la realidad se produce de las interacciones con el medio ambiente y que el conflicto cognitivo que se genera al enfrentar una nueva situación estimula el aprendizaje. Por otra parte, esta metodología activa el aprendizaje desde un entorno en el que las interacciones tienen lugar entre los miembros del propio grupo de estudiantes y entre éstos y el equipo docente, y finalmente, se retroalimenta constantemente al estudiante por parte del docente, tanto en las sesiones de evaluación como en el trato personal (Rué, Font y Cebrián, 2011, 27). El ABP promueve además, el autoaprendizaje, la adquisición de habilidades y actitudes, despertando el interés de los estudiantes al disfrutar del aprendizaje estimulando su creatividad y responsabilidad hacia problemas de su entorno más inmediato (Morales, 2007).

Para Pérez y Chamizo (2011), el $\mathrm{ABP}$ pretende proponer actividades que planteen situaciones problemáticas cuya resolución requiere analizar, descubrir, elaborar hipótesis, confrontar, reflexionar, argumentar y comunicar ideas para lograr el aprendizaje de los estudiantes. Para estos autores la resolución de problemas como estrategia implica utilizar problemas reales con preguntas auténticas que sean relevan- 
tes para los alumnos en el contexto del aprendizaje de ciencias, es decir, implica plantear preguntas como problemas. Sin embargo, plantear buenas preguntas no es fácil, y requiere de habilidad, práctica y tener conocimientos mínimos sobre el tema. Pérez y Chamizo (2011) señalan que para aplicar la estrategia del ABP en el ámbito educativo, es imprescindible establecer la definición de qué es un problema. Para ello, a pesar de las múltiples definiciones en torno al significado de "problema", los autores concluyen que éste debería cumplir tres condiciones: "una pregunta o cuestión, es decir, algo que no se sabe, algo por resolver; deseo, motivación, e interés en la resolución; y que suponga un reto, de forma que la estrategia de solución no resulte evidente".

La resolución de problemas ambientales como estrategia educativa ha sido empleada en experiencias previas tanto en la formación inicial del profesorado como en maestros/as en activo. Así lo constata el trabajo de Rivarosa y Perales (2006), quienes describen numerosas experiencias en ambos ámbitos llevadas a cabo en España y Argentina. En estudiantes de magisterio, Rivarosa y Perales (2006) plantean diversos problemas ambientales como el consumo, la contaminación atmosférica o el turismo y medio ambiente, entre otros. Por otro lado, en numerosas escuelas argentinas, las experiencias llevadas a cabo por maestros/as en activo se configuran en base a problemas ambientales locales y reales, muy acorde a considerar el medio ambiente en su totalidad, es decir, natural y creado por el hombre, ecológico, económico, tecnológico, social, legislativo, cultural y estético, un aspecto ya presente en los comienzos de la EA en la Conferencia Intergubernamental de Tbilisi (1977). En otras propuestas didácticas realizadas en la formación inicial del profesorado se plantean problemas más abiertos, como evaluar los efectos del Cambio Climático en los distintos niveles de organización de un ecosistema (Varela-Losada et al. 2014).

En este sentido, los problemas que se planteen desde el ABP deben permitir poner al estudiante en situación para aprender, de manera que, el alumno se sienta más involucrado, mostrando un mayor interés en la medida que identifique el problema como un reto y una posibilidad de aprendizaje significativo (Bejarano y Lirio, 2008). En nuestro caso, además se pretende que estos problemas propicien cambios significativos en los comportamientos medioambientales en los estudiantes. Sin embargo, no es fácil diferenciar y organizar problemáticas socionaturales reales y con sentido social (Rivarosa y Perales, 2006). Para lograr esto en la propuesta didáctica presentada en este trabajo se utiliza el HEU, que permite contextualizar la estrategia de ABP, abordando así algunas de las limitaciones que se han venido comentando hasta ahora. Por un lado, se conecta con un problema real, como es el estudio del suelo del huerto y por otro, la resolución del problema, conlleva una aplicación real, con acciones directas en el huerto, dado que el estudio del suelo es el primer paso 
para gestionar y poner en marcha dicho recurso.

\section{Metodología: Diseño y desarrollo de la estrategia de ABP}

La presente propuesta didáctica fue desarrollada desde la asignatura optativa de EAI, en el $4^{\circ}$ curso del Grado en Educación Infantil, en el marco de un proyecto de innovación y mejora docente, concedido por la Universidad de Cádiz en el curso 2014/2015. Dicha propuesta fue diseñada por los propios autores, $y$ participaron un total de 40 estudiantes, organizados en dos subgrupos correspondientes a las sesiones prácticas de la asignatura. La estrategia de ABP seguida fue similar en ambos subgrupos (salvo la sesión 4 que fue realizada en gran grupo), utilizando cinco sesiones de hora y media cada una.

Cada una de las etapas fue diseñada en base a diversos trabajos anteriores (Morales y Landa, 2004; De Miguel, 2006; Sola-Portolés, Sanjosé y Gómez, 2011; Esteban, 2011; Díaz-Garrido y Sánchez-López, 2015). La secuencia didáctica quedó estructurada tal y como se muestra en la tabla II. Los estudiantes, se organizaron en 9 equipos de trabajo de entre 5-6 componentes cada uno.

Para iniciar la estrategia se utilizó el HEU como escenario para conectar con el tema del suelo ofreciendo un contexto real en el que plantear un problema al que dar respuesta. El HEU permitía dar sentido al problema al estar relacionado directamente con una de las principales cuestiones a considerar a la hora de diseñar y gestionar un huerto, como son las características del suelo y la posibilidad de cultivar en él. Al inicio de cada etapa, se realizaba de forma breve, una presentación de cada una de las fases de la estrategia de ABP, con una doble intención; dar a conocer a los estudiantes la estrategia como contenido de la asignatura y establecer una relación directa entre la teoría y la práctica.

La sesión inicial (etapa 1), sirvió para introducir el tema y analizar el escenario del problema. Para ello, la docente planteó varias preguntas con objeto de propiciar el debate y una primera lluvia de ideas sobre los factores que consideran que intervienen en el crecimiento de las plantas, y la importancia del suelo como factor esencial para ello. Se realizó así, una primera exploración de sus ideas que fueron recogidas para su posterior análisis.

Para profundizar en sus concepciones previas y explicitar las ideas de los estudiantes sobre el concepto de suelo, de manera individual, los alumnos apuntaron su concepto de suelo para, posteriormente, confrontarla con los miembros de su equipo. Cada grupo elaboró su propia definición de suelo diseñando un mapa conceptual para establecer relaciones entre los diferentes conceptos sobre el suelo. Posteriormente, los grupos intercambiaron sus mapas conceptuales; cada uno valoró el mapa conceptual de otro grupo, señalando en él qué ideas compartían y cuáles no. Finalmente, cada grupo expuso 
brevemente los comentarios realizados sobre el mapa conceptual del grupo correspondiente contrastando así sus propias ideas.

En una segunda sesión (etapa 2 y 3), se pasó a definir el problema que los grupos debían dar solución; para ello se les planteó la pregunta: “¿qué tipo de suelo tiene nuestro huerto? ¿es cultivable?". A continuación, cada grupo diseñó su propio plan de acción en el que debían incluir aspectos como: qué técnicas emplearían para resolver dicho problema, qué material necesitarían, las fuentes bibliográficas consultadas y el reparto de tareas de cada componente del grupo. Cada plan de acción fue revisado por la docente con objeto de orientar el proceso de búsqueda de información, principalmente sobre las técnicas seleccionadas atendiendo a su viabilidad para su posterior desarrollo en el laboratorio. Entre las fuentes bibliográficas consultadas por los estudiantes se encontraron artículos científicos, páginas web de organismos oficiales, no oficiales y libros de diferentes autores. Respecto a los artículos utilizados, salvo uno, fueron de carácter científico (sobre edafología) más que didáctico. Por la temática y contenido de los mismos se aprecia que los estudiantes indagaron sobre cuestiones específicas del crecimiento de ciertos cultivos y las características físico-químicas del suelo. Las páginas web visitadas, en su mayoría, no pertenecían a ningún organismo oficial, desconociéndose el grado de análisis y verificación al que los alumnos sometieron la información recopilada en las mismas. Además, se consultaron blogs de agricultura y bloques temáticos elaborados por otros docentes, aunque sin vinculación aparente a ninguna institución de enseñanza o de divulgación científica. Por otro lado, se consultó y extrajo información de organismos como la FAO (Organización de las Naciones Unidas para la Alimentación y la Agricultura), el MAGRAMA (Ministerio de Agricultura, Alimentación y Medio Ambiente del Gobierno de España) y otras instituciones públicas de autonomías españolas como de gobiernos extranjeros como México y Argentina.

La tercera sesión (etapa 4) fue desarrollada en el laboratorio; los distintos grupos llevaron a cabo su plan de acción revisado por la docente, de manera totalmente autónoma. Cada uno, en base a las orientaciones realizadas por la docente, gestionó las técnicas y los procedimientos a seguir para dar respuesta al problema planteado. Algunas de las acciones que se desarrollaron fueron: medida del $\mathrm{pH}$ del suelo del huerto, estudio de la textura, la cantidad de materia orgánica y la presencia de seres vivos.

La cuarta sesión fue destinada a compartir en gran grupo los principales resultados obtenidos durante la fase de desarrollo, las conclusiones y soluciones de cada grupo al problema planteado (etapa 5). Cada grupo realizó una exposición oral de 5 minutos de duración. La docente, y los diferentes grupos valoraron la exposición de cada grupo, 
incluidos de ellos mismos mediante una rúbrica.

La quinta sesión (etapa 6) fue utilizada para confrontar sus propias ideas y revisar lo aprendido durante las etapas anteriores. La docente realizó una presentación de las etapas desarrolladas a través del ABP. Se comentaron los principales resultados obtenidos en la sesión anterior para resolver la pregunta planteada. También, se expusieron los contenidos específicos trabajados durante la estrategia: definición de suelo, características de un suelo para que sea cultivable, técnicas para conocer las características de un suelo, interpretación de algunos de sus resultados $(\mathrm{pH}$, cantidad de materia orgánica presente en la tierra del huerto, presencia de organismos vivos, etc.). Se amplió información y se resolvieron dudas surgidas durante las exposiciones grupales. Finalmente, se tomaron decisiones en función de los resultados obtenidos de las características del suelo y su idoneidad para el cultivo. Tras finalizar la sesión y evaluar su aprendizaje, haciendo uso de la plataforma del Campus Virtual de la asignatura, los estudiantes realizaron un cuestionario individual constituido por 12 preguntas de respuesta múltiple, con una sola respuesta correcta y 2 preguntas abiertas. Asimismo, cada grupo entregó un informe final de acuerdo a los criterios establecidos en la rúbrica diseñada para valorar dicho trabajo.

\section{Instrumentos para valorar la propuesta}

Para valorar la estrategia desarrollada se utilizaron algunos de los instrumentos señalados en cada etapa de la propuesta didáctica (tabla II). Concretamente, para este trabajo se ha utilizado la información obtenida a través de la dinámica inicial de lluvia de ideas, los mapas conceptuales grupales, el plan de acción y el cuestionario final individual.

Con los mapas conceptuales se realizó un análisis preliminar de las ideas previas de los alumnos sobre el concepto de suelo, tomando como referencia el trabajo de Murga-Menoyo (2011), prestando atención a los siguiente aspectos: a) Análisis de los conceptos con mayor frecuencia de aparición, b) Análisis de las relaciones realizadas e identificación de relaciones erróneas y c) Extracción de las ideas previas sobre el suelo y su categorización.

El plan de acción permitió valorar la diversidad de técnicas seleccionadas por cada grupo para resolver el problema planteado. En cuanto al cuestionario individual final, se analizaron las dos preguntas abiertas, referentes al concepto de suelo y a la percepción de los estudiantes sobre las competencias desarrolladas a través de la estrategia empleada.

\section{Resultados y discusión}

Algunas de las respuestas dadas por los estudiantes a través de la lluvia 


\section{Tabla II \\ Resumen de las etapas del ABP en la asignatura de EAI}

(Fuente: Elaboración propia)

\begin{tabular}{|c|c|c|}
\hline $\begin{array}{c}\text { Etapa de la } \\
\text { estrategia de ABP }\end{array}$ & Intenciones didácticas & $\begin{array}{c}\text { Instrumento de } \\
\text { evaluación }\end{array}$ \\
\hline $\begin{array}{l}\text { Análisis del escenario } \\
\text { problema }\end{array}$ & $\begin{array}{l}\text { Contextualizar el problema: el suelo como factor en } \\
\text { el crecimiento de las plantas } \\
\text { Explicitar las ideas previas de los estudiantes sobre } \\
\text { el concepto de suelo }\end{array}$ & $\begin{array}{l}\text { Lluvia de ideas } \\
\text { Mapa conceptual } \\
\text { grupal }\end{array}$ \\
\hline $\begin{array}{l}\text { Definición del } \\
\text { problema }\end{array}$ & $\begin{array}{l}\text { Planteamiento de la pregunta: ¿qué características } \\
\text { debe tener un suelo para que sea cultivable?, ¿cómo } \\
\text { es el suelo de nuestro huerto?, ¿es cultivable? }\end{array}$ & Portafolio grupal \\
\hline $\begin{array}{l}\text { Diseño del plan } \\
\text { de acción }\end{array}$ & $\begin{array}{l}\text { Cada grupo elabora su propio plan de acción de cara } \\
\text { al estudio de las características del suelo de nuestro } \\
\text { huerto. } \\
\text { Búsqueda de información para resolver el problema } \\
\text { planteado }\end{array}$ & $\begin{array}{l}\text { Esquema del plan de } \\
\text { acción }\end{array}$ \\
\hline $\begin{array}{l}\text { Desarrollo del plan } \\
\text { de acción }\end{array}$ & $\begin{array}{l}\text { En el laboratorio cada grupo pone en marcha las di- } \\
\text { ferentes técnicas y procedimientos según su plan de } \\
\text { acción para dar respuesta al problema planteado }\end{array}$ & Observación directa \\
\hline $\begin{array}{l}\text { Análisis de los } \\
\text { resultados y } \\
\text { obtención de } \\
\text { conclusiones }\end{array}$ & $\begin{array}{l}\text { Los grupos comparten sus soluciones y su trabajo } \\
\text { realizado al resto de la clase y las discuten. Cada gru- } \\
\text { po evalúa su exposición y la del resto de los grupos. }\end{array}$ & $\begin{array}{l}\text { Exposición grupal } \\
\text { Rúbrica para su } \\
\text { evaluación }\end{array}$ \\
\hline $\begin{array}{l}\text { Revisión final y } \\
\text { planteamiento del } \\
\text { nuevo interrogante }\end{array}$ & $\begin{array}{l}\text { Presentación por parte de la docente de cada una de } \\
\text { las etapas de la estrategia de ABP. } \\
\text { Revisión y resolución de cuestiones surgidas en la } \\
\text { sesión anterior. } \\
\text { Toma de decisiones sobre actuaciones a realizar en } \\
\text { el huerto en función de los resultados obtenidos des- } \\
\text { pués de la emisión de conclusiones } \\
\text { Relación del tema del suelo con el problema ambien- } \\
\text { tal de la erosión (inicio de la fase II de la estructura } \\
\text { de la asignatura) }\end{array}$ & $\begin{array}{l}\text { Cuestionario } \\
\text { individual final sobre } \\
\text { contenidos trabajados } \\
\text { y sobre la estrategia } \\
\text { Informe grupal, } \\
\text { con rúbrica para su } \\
\text { evaluación }\end{array}$ \\
\hline
\end{tabular}

de ideas en la etapa 1 eran términos relacionados con las necesidades de las plantas para crecer; en sus respuestas incluían ideas como la importancia de un clima específico para el crecimiento y de ciertos elementos claves como los minerales, el grado de humedad, el agua, la luz y la tierra. Los alumnos mostraron un cierto grado de conoci- miento sobre el tema, un aspecto fundamental, según indican Pérez y Chamizo (2011), para el planteamiento de "buenas preguntas" y poner en marcha la estrategia de $\mathrm{ABP}$, despertando su interés por querer saber más sobre lo que ya se conoce.

Para explorar sus ideas sobre el concepto de suelo se les formuló la pregun- 
ta “¿qué es el suelo?”, cuya respuesta debería ser consensuada en cada grupo y expresada a través de la elaboración de un mapa conceptual. Durante la sesión se observó que los estudiantes conocían muchos términos relacionados con el suelo, pero se detectaron algunas dificultades a la hora de realizar el mapa conceptual, así como en el uso de términos poco científicos. De esta primera aproximación, se pueden extraer los términos más frecuentes que manejan los estudiantes para definir qué es el suelo (tabla III). El término "superficie" apareció en 8 de los 9 mapas elaborados, el mapa conceptual restante utilizaba el término "base" y uno de los grupos utilizó ambos términos para referirse al suelo. Esta noción de suelo como "superficie" es común y forma parte del conocimiento cotidiano, siendo en ese contexto donde mantiene todo su sentido. Como señala Pedrinaci (1996), el problema de la concepción del término surge de su uso científico, donde presenta un significado distinto al del ámbito cotidiano. Esto no supone que exista un significado científico correcto y otro cotidiano incorrecto, sino teniendo cada uno de ellos un ámbito específico de aplicación. El término "agua" fue otro de los conceptos más repetidos, aunque ubicado en diferentes posiciones dentro de la jerarquía de los mapas. "Composición" y "componentes" son términos que se han incluido en 6 mapas, aunque el nivel jerárquico directamente inferior no ha incluido los mismos conceptos en cada uno de ellos.

\section{Tabla III \\ Resultados del análisis cualitativo de los mapas conceptuales en base a la frecuencia de aparición}

(Fuente: Elaboración propia)

\begin{tabular}{|l|c|}
\hline \multicolumn{1}{|c|}{ Términos relacionados con el concepto de suelo } & $\begin{array}{c}\text { Frecuencia (\%) de aparición } \\
\text { de los términos presentes en } \\
\text { los mapas conceptuales }\end{array}$ \\
\hline Superficie/base & 89 \\
Agua & 78 \\
Componentes/composición, minerales. & 67 \\
Rocas/piedras, tierra. & 56 \\
Restos de seres vivos, características, luz/iluminación. & 44 \\
Clima/climatología, elementos, humedad/húmedo, contaminación, \\
nutrientes, materia orgánica, natural, artificial.
\end{tabular}


Los conceptos más utilizados muestran cómo los alumnos relacionan de forma implícita el suelo con su función como soporte de la vida vegetal al describirlo en los mapas con términos como "agua", "minerales", "tierra", "luz", "nutrientes" y "materia orgánica”, aunque la vegetación o las plantas no se mencionan de forma explícita. Solo se registra en uno de los mapas la distinción entre "cultivable" y "no cultivable". De los términos cuantificados se extrae una concepción predominante sobre el carácter mineral del suelo y sus componentes, así como de los factores físico-químicos que afectan al mismo; no obstante, no se hacen referencias a procesos, sino a elementos aislados. La consideración de la fracción biótica del suelo se reduce a la introducción en dos de los mapas conceptuales del término "seres vivos" y "restos de seres vivos", "nutrientes" y "materia orgánica" en otros.

Un análisis inicial de las relaciones realizadas en los mapas conceptuales reveló una gran diversidad en el número de niveles jerárquicos incluidos, en el número y tipo de términos utilizados y en la ubicación de estos en el mapa. El número medio de niveles jerárquicos establecidos fue de 4. Partiendo del concepto "suelo" la primera relación establecida en todos los mapas fue con "superficie" y/o "base". Uno de los grupos no realizó esta identificación y estableció como primer nivel "tipos" de suelo. En este primer nivel, uno de los grupos realizó la división entre suelo "natural" y "edificado". En el segundo nivel, la mayoría de los grupos incluyeron términos como "características", "componentes" y "elementos" que se desarrollaban en el tercer nivel (incluido por todos los grupos excepto por uno). En el cuarto nivel realizan diversas referencias al clima y a los factores relacionados con el agua y la luz, utilizando términos como "agua, climatología, iluminación (natural y artificial), mano de obra humana, estaciones del año y contaminación."

El análisis general de los mapas conceptuales parece indicar que todos los grupos manejaban concepciones previas sobre el suelo muy próximas a la idea de "capa más superficial de la tierra, caracterizada por tener una estructura formada por una mezcla de restos minerales y materia orgánica". Los grupos apenas introducen en sus definiciones aspectos relacionados con los procesos físicos, químicos o biológicos que tienen lugar en él, probablemente por su mayor grado de abstracción y complejidad, ni hacen alusión a la vida vegetal que soporta. Por otro lado, ninguno presentó una visión dinámica asociada al concepto de suelo o relacionada con conceptos como, por ejemplo, el de ecosistema. Diversos estudios hacen alusión a dificultades e ideas previas que presentan los estudiantes respecto a las concepciones más comunes en actividades de EA (García 2004). Principalmente, el hecho de que los alumnos suelen reconocer sólo el mesocosmos, lo perceptible, con un gran desconocimiento de otros niveles de organización de la materia (microcosmos y macro- 
cosmos). Asimismo, los estudiantes sólo establecen relaciones de causa y efecto simples, obviando las relaciones bidireccionales entre los elementos de un sistema, y por último, la visión rígida y estática del mundo en todos los niveles de organización.

En relación a lo anterior, es interesante comparar estos resultados con el trabajo de Rebollo, Prieto y Brero (2005), basado en investigaciones anteriores de Happs (1984) y Yus y Rebollo (1993), que establecen un paralelismo entre las concepciones de los estudiantes y las ideas sostenidas por los científicos en el pasado, partiendo de la historia y epistemología de la ciencia del estudio del suelo. En este trabajo, se encuadra a los alumnos (de 12 a 17 años) en los primeros estadios de la evolución de dicha ciencia, bajo un paradigma agrológico que consideraría el suelo únicamente como soporte de la vida vegetal. Aproximan- do nuestros resultados a la clasificación realizada por Rebollo et al. (2005), encontramos que las concepciones mostradas por nuestros alumnos se considerarían cercanas a las visiones agrológica y química (tabla IV), que se corresponden con paradigmas de los primeros estadios de la historia del estudio del suelo. Dichas visiones se enfocan de manera general a la concepción del suelo únicamente como soporte de la vida vegetal y al estudio de la composición del suelo a partir de la utilización de métodos analíticos. Los términos utilizados en los mapas conceptuales (analizados en base a la tabla III) y las experiencias realizadas en el laboratorio pueden comprenderse bajo este paradigma y no tanto bajo la visión geológica, puesto que no se atienden aspectos de transformación mecánica $o$ disgregación de rocas para la formación del suelo ni procesos edáficos en general.

\section{Tabla IV \\ Evolución del concepto de suelo en diferentes etapas y descripción por sus componentes más relevantes}

(Fuente: modificado de Rebollo et al., 2005)

\begin{tabular}{|l|l|l|}
\hline \multicolumn{1}{c}{$\begin{array}{c}\text { Concepción } \\
\text { epistemológica }\end{array}$} & \multicolumn{2}{c|}{ Visión científica } \\
\hline \multirow{2}{*}{ Estática } & 1. Agrológica & El suelo como simple soporte de la vida vegetal. \\
\cline { 2 - 3 } & 2. Química & $\begin{array}{l}\text { El suelo definido por su composición, asociada al } \\
\text { empleo de métodos analíticos. }\end{array}$ \\
\cline { 2 - 3 } & 3. Geológica & El suelo como producto de alteración de las rocas. \\
\hline $\begin{array}{l}\text { Nacimiento de la } \\
\text { edafología (1880-1890) }\end{array}$ & 4. Interdisciplinar & El suelo como un ente natural. \\
\hline Dinámica & Ecológica & $\begin{array}{l}\text { El suelo como ecosistema; interrelación y } \\
\text { dinamismo. }\end{array}$ \\
\hline
\end{tabular}


La revisión de los planes de acción revela que los estudiantes fueron capaces de organizar, plantear y llevar a cabo un plan de trabajo adecuado para resolver el problema tratado y las técnicas y metodologías seleccionadas fueron muy diversas. Los nueve grupos midieron el $\mathrm{pH}$ del suelo utilizando papel de tornasol (uno de ellos, además, utilizó el agua obtenida de la cocción de una col); siete analizaron la textura del suelo, bien tamizando o bien por gravimetría; siete estudiaron el contenido en materia orgánica del suelo con técnicas basadas en la reacción entre una muestra de suelo y agua oxigenada; tres de ellos utilizaron la presencia de seres vivos como indicador de fertilidad y uno utilizó el estudio de las plantas del huerto para conocer las caracterís- ticas del suelo. La docente sólo asesoró a los grupos atendiendo al grado de viabilidad de las técnicas propuestas y material necesario para cada una de ellas en el laboratorio. Los grupos desarrollaron de forma autónoma su plan de acción, registrando todo el proceso a través de imágenes para la elaboración de un informe final.

El cuestionario final fue realizado por 38 de los 40 alumnos; sus concepciones se agruparon en tres categorías atendiendo a diferentes grados de complejidad respecto al concepto de suelo (gráfico 1). El 57,9\% de los alumnos mantenían una concepción caracterizada por la enumeración de elementos componentes del suelo y la presencia de relaciones sencillas entre dichos elementos, muy cercana a la idea inicial

\section{Gráfico I}

Concepciones alternativas mostradas en los estudiantes de EAI

(Fuente: Elaboración propia)

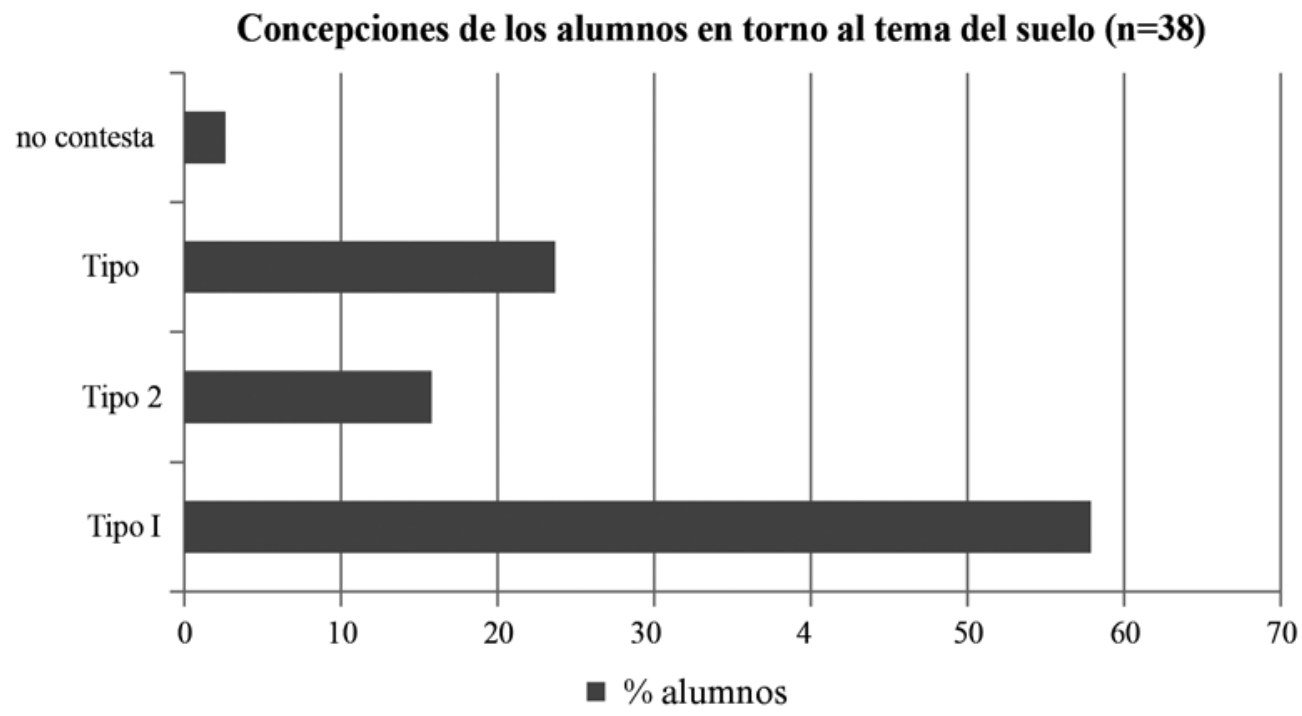




\section{Gráfico II \\ Competencias desarrolladas por los alumnos de EAI}

(Fuente: Elaboración propia)

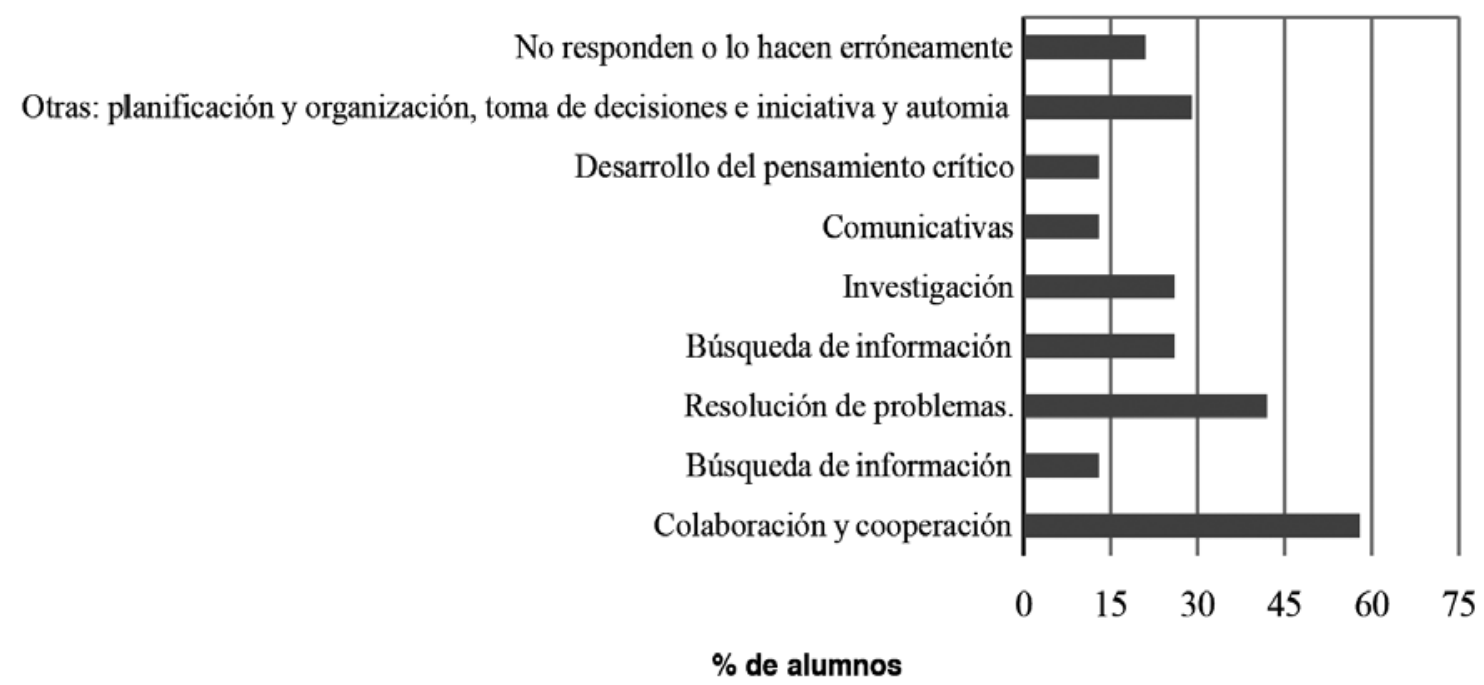

reflejada en los mapas conceptuales. El $18,1 \%$, ofreció una concepción en la que se mostraban relaciones entre los diferentes elementos, aunque en la mayoría de sus concepciones predominaba una relación lineal y mecánica. Finalmente, el $21 \%$ de los estudiantes mostró una idea de suelo como algo vivo y cambiante, aunque aún predomina la concepción de relación lineal causa-efecto.

Cuando se preguntó a los alumnos por las competencias profesionales que creían haber adquirido durante la estrategia (gráfico 2). Más de la mitad señalaron la capacidad de colaborar y cooperar entre ellos y la capacidad de resolver problemas como las competen- cias más desarrolladas, un resultado que coincide con el trabajo de GómezEsquer et al., (2009). Existen además, coincidencias en las competencias menos desarrolladas, como es el razonamiento crítico. Si bien, hay que indicar que en este estudio, a diferencia del realizado por los autores anteriores, en el cuestionario final se les dio total libertad a la hora de indicar sus opiniones, lo que podría explicar el porcentaje de estudiantes, $21 \%$, que no contestaron a esta pregunta o bien contestaron de forma errónea. Sin duda, será un aspecto a tener en cuenta en futuros estudios. 


\section{Conclusiones finales}

Los resultados obtenidos en el presente trabajo parecen indicar que la estrategia de $\mathrm{ABP}$ puede ser muy útil para el desarrollo de las competencias que se indican desde el EEES, así como las que se establecen en la asignatura de EAI. Nuestro objetivo principal es formar a futuros maestros/as con compromiso ambiental, a la vez, que estos sean capaces de diseñar y desarrollar propuestas didácticas acorde a una EA para la acción a través de enfoques constructivistas como el ABP y el planteamiento de problemas ambientales que impliquen una educación integral de los niños y niñas de la etapa de infantil.

Asimismo, el ABP junto al uso del HEU como contexto de aprendizaje en la formación inicial de maestros/as, presenta numerosas ventajas; por un lado, logra despertar el interés de los estudiantes, su curiosidad por aprender y, sobre todo, la sensación de ser protagonistas de su propio aprendizaje. La estrategia contribuye a la construcción de conocimientos en torno al tema del suelo, conceptuales como procedimentales, principalmente a la hora de reproducir técnicas y metodologías que conllevan cierto conocimiento y manejo del material de laboratorio. En cuanto a las actitudes, los estudiantes mostraron un elevado interés hacia el tema, dado que el problema planteado suponía el primer paso para trabajar el HEU. Por otro lado, el hecho de realizar algunas de las sesiones en el laboratorio supu- so gran expectación entre el alumnado, debido al escaso uso que se hace de este espacio durante su formación universitaria, aspecto que será considerado en futuras acciones. No obstante, también se han observado ciertas limitaciones e inconvenientes como constatan trabajos anteriores, como es la sensación de desconcierto, incertidumbre e inseguridad que existe entre los estudiantes (Egido et al., 2007; Solaz-Portolés et al., 2011), sobre todo en las primeras etapas de la estrategia. Asimismo, tras la puesta en marcha de esta estrategia, somos conscientes de otras limitaciones que surgen, como es la demanda de recursos humanos y materiales que pueden llegar a ser muy costosos, tanto a nivel económico como de tiempo.

Otras experiencias realizadas en torno al ABP como estrategia para la EA en estudiantes de magisterio coinciden con algunas de las conclusiones obtenidas en nuestro trabajo. ValeraLosada et al. (2014) indican que los futuros maestros/as valoran positivamente la implementación de metodologías más participativas como el $\mathrm{ABP}$ para la EA favoreciendo su aprendizaje, no solo en conocimientos vinculados con este ámbito, como el de ecosistema, sino también, a la hora de comprender un problema ambiental complejo. Si bien, detectan ciertas carencias que serían interesantes atender para desarrollar este tipo de estrategias. Por un lado, los escasos conocimientos que presentan los estudiantes sobre el medio ambiente y su dificultad sobre ciertas competencias genéricas relacionadas con la 
búsqueda de información, la selección y el análisis de información. Por otro, en algunos casos, no llegan a interiorizar la importancia de usar metodologías activas en la enseñanza de la EA, por lo que conviene hacer un análisis reflexivo de las propuestas llevadas a cabo.

Utilizar el HEU como medio para responder a cuestiones como el tipo de suelo que tiene nuestro huerto presenta un enorme potencial didáctico, no solo por la diversidad de conocimientos que se ponen en juego a la hora de enfocar el problema y dar solución a éste, sino que permite dotar de significado y funcionalidad a los nuevos aprendizajes. De hecho, tras compartir las aportaciones realizadas por los diferentes grupos, se concluyó que nuestro suelo era pobre en materia orgánica, con textura arcillo-arenoso y con escasa presencia de organismos vivos. Tras la revisión y puesta en común del proceso, se tomó la decisión conjunta de abonar la tierra antes de comenzar con el diseño y el desarrollo del HEU en base a los estudios realizados por los diferentes grupos. El huerto junto a la estrategia de ABP permite trasladar los aprendizajes conseguidos y conectar así, con el estudio y análisis de algunos problemas ambientales en la siguiente fase de la asignatura. Para ello sería conveniente seleccionar problemas ambientales que permitan conectar estos directamente con el huerto, para poder profundizar sobre dichos problemas ambientales y buscar alternativas que conlleven acciones factibles en el huerto.
La asignatura de EAI se estructura en varias fases que se conectan entre sí hasta el punto que en las fases I y II se pretenden que los estudiantes conozcan y vivan nuevas estrategias y recursos alternativos útiles en el contexto de la EA aplicables en su futuro profesional. En este sentido, la fase I juega un papel esencial principalmente a la hora de diseñar futuras propuestas didácticas en torno a problemas ambientales en la etapa de infantil que deberán realizar en la fase III. El reto para nosotros, se sitúa en diseñar y articular la asignatura en torno a un recurso como el HEU, orientada a una EA que permita generar aprendizajes transferibles a la escuela con implicaciones metodológicas en sus prácticas docentes futuras. Para esto, conviene no sólo desarrollar experiencias en torno a nuevas estrategias, también se debe reflexionar sobre ellas y discutir de manera explícita con el alumnado las implicaciones didácticas, sus ventajas e inconvenientes. $\mathrm{Al}$ igual que ocurre en el ámbito de la EA, no se trata de convencer para propiciar cambios, se trata de negociar.

\section{Referencias bibliográficas}

BEJARANO, M.T., y LIRIO, J. (2008). La utilización de problemas auténticos en la Enseñanza Superior. En A. ESCRIBANO y A. DEL VALLE (coords.), El Aprendizaje basado en problemas. Una propuesta metodológica en Educación Superior. Madrid. Narcea. 
DE MIGUEL, M. (coord.) (2006). Modalidades de enseñanza centradas en el desarrollo de competencias. Orientaciones para el profesorado universitario ante el Espacio Europeo de Educación Superior. Madrid: Alianza editorial. Recuperado de http://www. unizar.es/ice/images/stories/materiales/ea2005-0118.pdf [20 de junio de 20141

EGIDO, I., et al. (2007). El Aprendizaje Basado en Problemas como innovación docente en la universidad: posibilidades y limitaciones. Educación y Futuro, no 16, 85-100.

ESTEBAN, M. (2011). Del "Aprendizaje Basado en Problemas" (ABP) al "Aprendizaje Basado En La Acción" (ABA). Claves para su complementariedad e implementación. Revista de Docencia Universitaria, Vol. 9 (1), Enero-Abril, 91-107.

GARCÍA, J.E. (2002). Los problemas de la Educación Ambiental: ¿Es posible una Educación Ambiental Integradora? Investigación en la Escuela, $\mathrm{n}^{\circ} 46$, 5-25.

GARCÍA, J.E. (2004). Educación Ambiental, Constructivismo y Complejidad. Sevilla: Diada.

GÓMEZ-ESQUER, F.; RIVAS, I.; MERCADO, F. y BARJOLA, P. (2009). Aplicación interdisciplinar del Aprendizaje Basado en Problemas (ABP) en Ciencias de la Salud: Una herramienta útil para el desarrollo de competencias profesionales. Revista de Docencia Universitaria, vol. 7 (4), 1-19.
GONZÁLEZ, M.T., y CASTRO, A. (2011). Impacto del ABP en el Desarrollo de la Habilidad para Formular Preguntas de Aprendizaje en Estudiantes Universitarios. Revista de Docencia Universitaria, vol. 9 (1), 57-66.

HAPPS, J.C. (1984). The utility of alternative knowledge frameworks in effecting conceptual change: some examples from the earth sciences. Tesis doctoral. Universidad de Waikato.

MARTÍN-PEÑA，M.L.; DÍAZGARRIDO, E. y SÁNCHEZ-LÓPEZ, JM. (2015). Coordinación interdisciplinar mediante aprendizaje basado en problemas. Una aplicación en las asignaturas dirección de producción y estadística empresarial. Revista de Investigación Educativa, 33(1), 163178. DOI: http://dx.doi.org/10.6018/ rie.33.1.179741

MANZANARES, A. (2008). Sobre el Aprendizaje Basado en Problemas (ABP). En A. ESCRIBANO y A. DEL VALLE, A. (coords), El Aprendizaje basado en problemas. Una propuesta metodológica en Educación Superior. Madrid: Narcea.

MORALES, M.L. (2008). Empleo del Aprendizaje Basado en Problemas (ABP). Una propuesta para acercarse a la química verde. Tecnología en Marcha, Vol. 21-1, 41-48.

MORALES, P. y LANDA, V. (2004). Aprendizaje basado en problemas. Theoria, Vol.13, 145-157. Recuperado de http://redalyc.uaemex.mx/ redalyc/pdf/299/29901314.pdf 
MURGA-MENOYO, M.A; BAUTISTACERRO, M.J. y NOVO, M. (2011). Mapas Conceptuales con cmap tools en la enseñanza universitaria de la educación ambiental. Estudio de caso en la UNED. Enseñanza de las Ciencias, 29(1), 47-60.

PEDRINACI, E. (1996). Sobre la persistencia o no de las ideas del alumnado en geología. Alambique: Didáctica de las Ciencias Experimentales, $\mathrm{n}^{\mathrm{0}} 7$, 27-36.

PÉREZ, Y. y CHAMIZO, J.A. (2011). Los museos: un instrumento para el Aprendizaje Basado en Problemas (ABP). Revista Eureka sobre Enseñanza y Divulgación de las Ciencias, 8 (3), 312-322.

REBOLLO, M.; PRIETO, T. y BRERO, V. (2005). Aproximación a la historia epistemológica del concepto de suelo: implicaciones didácticas. Enseñanza de las Ciencias. ${ }^{\circ}$ Extra, VII Congreso Internacional sobre Investigación en la Didáctica de las Ciencias.

RIVAROSA, A., y PERALES, F.J. (2006). La resolución de problemas ambientales en la escuela y en la formación inicial de maestros. Revista Iberoamericana de Educación, nº 40, 111-124.

RUÉ, J.; FONT, A., Y CEBRIÁN, G. (2011). El ABP, un enfoque estratégico para la formación en Educación Superior, Aportaciones de un análisis de la formación en Derecho. Revista de Docencia Universitaria, vol. 9 (1), 25-44.

SÁNCHEZ, I. y RAMIS, F.J., (2004). Aprendizaje significativo basado en problemas. Horizontes Educacionales, $\mathrm{n}^{\circ}$ 9, 101-111. Recuperado de: http://www.redalyc.org/ pdf/979/97917171011.pdf

SOLAZ-PORTOLÉS, J.J.; SANJOSÉ, V., y GÓMEZ, A. (2011). Aprendizaje basado en problemas en la Educación Superior. Una metodología necesaria en la formación del profesorado. Didáctica de las Ciencias Experimentales y Sociales, $\mathrm{n}^{\circ} 25,177-186$.

UNESCO (1980). La educación ambiental. Las grandes orientaciones de la Conferencia de Tbilisi. París: UNESCO.

VARELA-LOSADA, M.; PÉREZRODRÍGUEZ, U.; ÁLVAREZ-LIRES, F.J. y ÁLVAREZ-LIRES, M.M. (2014). Desarrollo de Competencias Docentes a partir de Metodologías Participativas Aplicadas a la Educación Ambiental. Formación Universitaria, vol. 7(6), 27-36. DOI: 10.4067/S071850062014000600004

YUS, R. y REBOLLO, M. (1993). Aproximación a los problemas de aprendizaje de la estructura y formación del suelo en el alumnado de 12 a 17 años. Enseñanza de las Ciencias, 11 (3), 265-280. 